\title{
8. Vous les entendez ? Analyse du discours et dialogisme
}

Jacques Bres

\section{CpenEdition \\ Journals}

Édition électronique

URL : https://journals.openedition.org/ml/1411

DOI : $10.4000 / \mathrm{ml} .1411$

ISSN : 2274-0511

Éditeur

Association Modèles linguistiques

Édition imprimée

Date de publication : 1 juillet 1999

Pagination : 71-86

Référence électronique

Jacques Bres, «8. Vous les entendez? Analyse du discours et dialogisme », Modèles linguistiques [En ligne], 40 | 1999, mis en ligne le 01 mai 2017, consulté le 01 juillet 2021. URL : http://

journals.openedition.org/ml/1411; DOI : https://doi.org/10.4000/ml.1411

Ce document a été généré automatiquement le 1 juillet 2021.

(C) Modèles Linguistiques 


\title{
8. Vous les entendez? Analyse du discours et dialogisme
}

\author{
Jacques Bres
}

1 L'expression d'un énoncé est toujours, à des degrés divers, une réponse, autrement dit : elle manifeste non seulement son propre rapport à l'objet de l'énoncé, mais aussi le rapport du locuteur aux énoncés d'autrui. Les formes de réactions-réponses qui remplissent un énoncé sont extraordinairement variées et, jusqu'à présent, elles n'ont jamais été étudiées (Bakhtine 1979/1984, p. 299).

2 Un des concepts essentiels de l'analyse de discours en France autour des années soixante-dix est assurément celui d'interdiscours, dont une approche historique devra analyser la place, l'économie, les espoirs dont il était porteur, les déconvenues dont il a été le lieu... Tel ne sera pas l'objet de la présente communication qui fait travailler pratiquement un concept venu de l'horizon théorique à la fois proche et lointain des recherches bakhtiniennes ${ }^{1}$ : le dialogisme.

3 Selon Bakhtine, la réalité langagière de la langue en discours, c'est le dialogue sous sa double forme de (i) dialogue externe ou dimension dialogale, que marque l'alternance des tours de parole référés à des énonciateurs différents et de (ii) dialogue interne, ou dimension dialogique, que réalisent tant les genres monologaux que les genres dialogaux. Le dialogisme est cette dimension constitutive qui tient à ce que le discours ne peut pas ne pas se réaliser dans un dialogue implicite avec d'autres discours et ce doublement ${ }^{2}$, interdiscursivement et interlocutivement: "Se constituant dans l'atmosphère du déjà-dit, le discours est déterminé en même temps par la réplique non encore dite, mais sollicitée et déjà prévue » $(1934 / 1978$, p. 103). Tout énoncé apparaît dans sa (joyeuse) incomplétude comme hétérogénéité énonciative, qui fait signe vers d'autres énoncés et invite à le replacer dans les dialogues internes (et, pour les genres dialogaux, externes) qui présidèrent à sa production, et peuvent seuls rendre compte de sa structure.

4 Si la production discursive se réalise dans la rencontre des discours autres sur le même thème (dialogisme interdiscursif, mémoire) et du discours-réponse de l'énonciataire (dialogisme interlocutif, par anticipation), quelles sont les traces, au niveau du discours 
produit, de cette double interaction, qui seules peuvent permettre d'étudier linguistiquement le dialogisme?

5 Lesdites traces sont nombreuses, leurs signifiants hétérogènes, leur mode d'être très variable (de l'explicite clairement marqué à l'implicite laissé à l'interprétation) ${ }^{3}$. J'en décrirai, de façon forcément superficielle, quelques-unes ${ }^{4}$ - conditionnel, négation, comparaison, renchérissement, confirmation, concession, opposition, subordination en appui sur un corpus d'articles de presse écrite dialogiques (éditorial, point de vue, débats).

Soit pour commencer, rapidement brossé, le cadre théorique suivant : reprenant à Bally (1934/1965, p. 36-38) l'analyse de l'actualisation phrastique comme application d'un modus à un dictum et la distinction entre sujet modal et sujet parlant (que je nommerai respectivement énonciateur et locuteur), je dirai que l'énonciateur (E1) modalise un dictum - le transformant de ce fait en un énoncé actualisé (E) - pour l'énonciataire (E2). J'appellerai dialogique un énoncé (ou fragment d'énoncé) dans lequel la modalisation de E1 s'applique à un dictum présenté comme ayant déjà statut d'énoncé (soit e), c'est-àdire ayant fait l'objet d'une modalisation par un autre énonciateur, que je désigne par e1. Illustrons ce propos par l'analyse de l'énoncé E suivant (titre d'article) :

(1) Oui, la quête d'Aurore Brossard est légitime

7 L'énonciateur E1 attribue l'assertion de l'énoncé e [la quête d'Aurore Brossard est légitime] à un énonciateur e1 et se charge de la confirmer par l'adverbe oui. Le dialogisme est analysable comme dédoublement énonciatif - deux énoncés ( $\mathrm{E}$ et e), deux énonciateurs (E1 et e1), deux énonciataires (E2 et e2) et, au-delà, deux systèmes de coordonnées spatio-temporelles - signifié (le plus souvent) par un marqueur de dialogisation (ici l'adverbe de confirmation oui). Précisons que le dédoublement énonciatif pose les éléments non pas à égalité mais hiérarchiquement, ce qu'essaie de noter la différence de caractères typographiques: $\mathrm{E}$ domine syntaxiquement $\mathrm{e}$ (enchâssement $\mathrm{E}(\mathrm{e})$ ), comme E1 domine énonciativement e1 (E1(e1)). E1 et e1 peuvent être co-référentiels $(\mathrm{E} 1=\mathrm{e} 1$, auto-dialogisme), être inclus l'un dans l'autre $(\mathrm{E} 1 \subset \mathrm{e} 1)$, ou référer à des entités différentes $(\mathrm{E} 1 \neq \mathrm{e} 1)$. Nous nous intéresserons ici à ce seul dernier cas.

\section{Le conditionnel}

8 La grammaire traditionnelle parle de conditionnel journalistique pour l'emploi dialogique de cette forme verbale qui a notamment fait l'objet de plusieurs études convergentes avec la position que je développe (Adamson 1994, Vion 1994, Haillet 1995 et 1997). Soit :

(2) Ce genre musical (le rap) [...] ne serait rien d'autre qu'une vaste entreprise de déstabilisation des valeurs morales.

Le conditionnel dédouble l'instance énonciative en e1 à qui est attribué l'assertion [Ce genre musical [...] n'est rien d'autre qu'une vaste entreprise de déstabilisation des valeurs morales.] et E1 qui, du fait du conditionnel, est présenté citant cette assertion mais ne se prononçant pas sur sa valeur de vérité, ce qui peut produire des effets de sens variés selon le co(n)-texte : simple suspension de la validation, doute, rejet...

Comment expliquer que le conditionnel (forme simple aussi bien que forme composée) puisse réaliser un dédoublement énonciatif? À la différence du futur qui s'appuie sur 
du présent (ce ser-a), en conquête de réalité, le conditionnel s'appuie sur la terminaison décadente de l'imparfait (ce ser-ait), en déprise de réalité. Le décalage temporel (présent > conditionnel), soit le passage de la conquête à la fuite de la réalité, vaut pour le décalage énonciatif $\mathrm{E} 1>\mathrm{e} 1^{5}$.

\section{La négation}

11 La linguistique contemporaine, en se passionnant pour les vertus dialogiques de cet outil (entre autres : Ducrot 1980, p. 49-56, 1984, p. 216-223, Berrendonner 1981, p 54-58 : « négation polémique »; Danon-Boileau 1987 : « négation modale »), retrouve - parfois sans le savoir - les analyses développées par Bergson 1907 : « Si je dis « cette table n'est pas blanche» [...] ce n'est pas sur la table elle-même que je porte ce jugement, mais plutôt sur le jugement qui la déclarerait blanche" (L'Evolution créatrice, cité par Grevisse, Le bon usage, p. 127). Soit :

(3) Je ne détourne pas mon regard de la réalité.

12 L'énonciateur E1 met en scène un énonciateur e1 à qui il attribue l'assertion de l'énoncé positif [Vous détournez votre regard de la réalité.], énoncé auquel il s'oppose par la négation. En (3), le co-texte indique que e1 correspond à l'énonciataire E2. E1 vient de décrire autrement (que ne le fait le discours dominant) la banlieue: il répond par avance à l'objection de naïveté qui pourrait lui être opposée. Le dédoublement énonciatif produit par la négation dialogique se vérifie de ce que (3) est paraphrasable $\operatorname{par}(3 a)$ :

(3a) Je ne pense pas que je détourne mon regard de la réalité.

qui sépare la modalisation négative de E1 (proposition principale) de l'assertion positive de e1 (subordonnée). Le fonctionnement réfutatif est parfois souligné par l'adverbe non, précédant l'énoncé négatif, qui introduit une marque de dialogalité dans un texte monologal :

(4) Non, les jeunes de La Paillade ne sont pas tous des drogués et des voleurs.

L'identité de e1 est parfois précisée, notamment par le tour contrairement à ce que dit $X$ :

(5) Contrairement à ce qu'il [El Kabbache] écrit, j'affirme qu'aucun de ces six animateurs n'a fait l'objet d'offres supérieures.

La présence de l'énoncé de e1 sous/dans celui de E1 s'explicite parfois par :

- Un autre marqueur dialogique (les guillemets en (6), le conditionnel en (7)) :

(6) Boris Elstine et ses proches collaborateurs [...] en envoyant l'armée en Tchétchénie, ne se sont pas inquiétés du «maintien de l'intégrité de la Fédération de Russie ».

(7) Aujourd'hui en Russie, au pouvoir, il n'y a pas de réformateurs qui seraient dans l'erreur.

- Un enchaînement co-textuel sur l'énoncé de e1 et non sur celui de E1 :

(8) L'intérêt de la France et l'intérêt de l'Allemagne ne sont pas antagonistes. Il y a eu un temps où on pouvait l'affirmer. [...]

Le pronom le anaphorise l'énoncé positif de e1: [L'intérêt de la France et l'intérêt de l'Allemagne sont antagonistes], et non sa réfutation par E1.

19

Dans la négation, E1 nie l'énoncé de e1. Ce qui est affirmé au-delà peut rester dans l'implicite parce que facilement inférable ou être développé par le co-texte ultérieur. 
On note que cet élément, lorsqu'il est explicité, est fréquemment introduit par mais. Soit [... Vnég + $\mathrm{x}$ mais $\mathrm{y}]$ :

(9) La liquidation de l'URSS n'a pas conduit à une amélioration du bien-être des

Russes mais à leur paupérisation.

La rectification marquée par mais s'effectue, en relais de la négation qui précède, sur l'énoncé de $\mathrm{e} 1$, à savoir $\mathrm{x}$.

La négation pose la relation E1/e1 comme agonale : E1 rejette comme fausse l'assertion de e1. Elle est l'outil parfait pour polémiquer avec l'autre.

Terminons ces remarques par la rapide description de deux tours négatifs :

- Au-delà du tour précédent réalisé en (9), on trouve - la portée de la négation ne s'effectuant plus que sur le complément - la structure [... V + non x mais y] :

(10) Il est temps de tourner le dos, non à des règles dont nous nous sommes par trop écartés, mais à des pratiques qui les ont dévoyées.

24 Cet énoncé est analysable, du point de vue de la dynamique communicative, en thème [il est temps de tourner le dos] + rhème1 rejeté [non à des règles dont nous nous sommes par trop écartés] + rhème 2 posé [mais à des pratiques qui les ont dévoyées]. Le fonctionnement dialogique est ici plus complexe : l'énoncé de e1 correspond à : [Il est temps de tourner le dos à des règles dont nous nous sommes par trop écartés.]; E1 le reprend pour s'accorder avec sa première partie (qui se trouve thématisée) et rejeter la seconde ( $\mathrm{x}$, rhème 1 rejeté). La voix de l'autre résonne doublement: E1 converge d'abord avec elle (sur le thème) pour mieux diverger ensuite d'elle (sur le rhème). On retrouve ce même mouvement dialogique avec l'extraction (Bres 1998).

- La négation restrictive (ne... que) pose d'intéressants problèmes de dialogisme qui ne semblent pas avoir arrêté les chercheurs. Soit :

(11) Quand l'existence [des migrants] est mise en question à sa racine, l'interprétation culturaliste [de leurs souffrances psychiques] n'est qu'un plâtrage sur une blessure à vif.

Co-texte : l'énonciateur de l'article conteste le bien-fondé de l'« orthopédie ethnique » développée par un de ses collègues psychiatres pour traiter les blessures psychiques des migrants. Comparons (11) à :

(11a) l'interprétation culturaliste est un plâtrage sur une blessure à vif.

(11b) l'interprétation culturaliste est non une thérapie efficace mais un plâtrage sur une blessure à vif.

En (11a), assertion sans négation, les échos dialogiques ont disparu: plus d'e1 sous E1. En (11b), l'énoncé de e1 est mentionné (rhème non $x$ ). La négation restrictive (11) sert à rejeter, sans les mentionner mais en les convoquant dans le geste même de ce rejet, les rhèmes que différents énonciateurs e1 (le tiers à qui E1 s'oppose mais aussi peut-être l'énonciataire E2) ont proposé ou peuvent proposer. La restriction laisse entendre d'autres voix mais ne cite pas leur énoncé. Elle apparaît comme plus agonale que la négation en ne... pas: E1 ne prend même pas la peine de rapporter l'énoncé de e $1^{6}$.

\section{Comparaison par corrélation}

Certains tours comparatifs, qui mettent en relation deux éléments argumentatifs, sont d'excellents candidats au marquage de l'altérité énonciative. Distinguons les comparaisons d'inégalité et d'égalité : 


\subsection{Comparaisons d'inégalité}

L'inégalité peut être de supériorité ou d'infériorité :

- Comparaison de supériorité : [... plus / davantage x que y] :

(12) La croissance britannique [...] relève davantage de son appartenance au monde anglo-américain [...] que de la valeur de sa monnaie.

Sur le thème [la croissance britannique relève de], E1 met en relation deux rhèmes $\mathrm{x}$ et y pour déclarer la supériorité de la pertinence argumentative du premier [son appartenance au monde anglo-américain], qu'il s'attribue, sur le second [la valeur de sa monnaie], qu'il attribue à e1.

- Comparaison d'infériorité : [... moins x que y] :

(13) La spécificité de l'époque actuelle réside moins dans la critique de la police que dans la façon dont celle-ci est formulée.

Sur le thème [la spécificité de l'époque actuelle réside dans], E1 met en relation deux rhèmes $\mathrm{x}$ et $\mathrm{y}$ pour déclarer l'infériorité de la pertinence argumentative du premier [la critique de la police] qu'il attribue à e1 sur le second [la façon dont celle-ci est formulée] qu'il s'attribue.

Comment rendre compte de ce chiasme énonciatif selon lequel dans la comparaison de supériorité $\mathrm{E} 1$ est inscrit sous $\mathrm{x}$ et e1 sous $\mathrm{y}$, alors que, dans la comparaison d'infériorité, e1 est inscrit sous $\mathrm{x}$ et $\mathrm{E} 1$ sous $\mathrm{y}$ ? L'explication me semble relever non du linguistique mais du discursif: selon le principe de valorisation du même / dévalorisation de l'autre (Bres 1993 : 139), E1 s'inscrit sous l'argument de plus grande pertinence et inscrit l'autre (e1) sous l'argument de moindre pertinence ${ }^{7}$. Que se passet-il lorsque les deux arguments sont posés à égalité de pertinence?

\subsection{Comparaison d'égalité}

Soit [autant x que y] :

(14) La rhétorique familiale se trouve autant dans la littérature de la Résistance que dans celle de Vichy.

Selon l'analyse précédente, on dira que, sur le thème [la rhétorique familiale se trouve], $\mathrm{E} 1$ met en relation deux rhèmes $\mathrm{x}$ et $\mathrm{y}$ pour déclarer l'égalité de la pertinence argumentative du premier (dans la littérature de la Résistance) et du second (dans celle de Vichy). Mais comment rendre compte ici de ce que E1 s'attribue x et attribue y à e1, dans la mesure où le principe de valorisation du même/dévalorisation de l'autre ne peut jouer puisque les deux rhèmes sont déclarés à égalité de pertinence ? La solution, en modification de l'analyse avancée jusqu'à présent, pourrait être la suivante: le terme comparé, $\mathrm{x}$, est d'une rhématicité supérieure à celle du terme comparant, $\mathrm{y}$, qui, en tant qu'étalon, fait partie du déjà dit ${ }^{8}$. Dans le cas de la comparaison d'égalité ou de supériorité, la parole de E1 en tant qu'elle prétend apporter du nouveau s'investit sous $\mathrm{x}$, la parole de l'autre (e1) s'inscrivant en déjà dit (y), en accord avec la différence de rhématicité de $\mathrm{x}$ et de $\mathrm{y}^{9}$. Dans le cas de la comparaison d'infériorité, le principe de valorisation / dévalorisation s'avère plus fort que la différence de rhématicité de $\mathrm{x}$ et de $y$, et E1 s'inscrit sous y et inscrit e1 sous $x$. 
évaluer la façon dont la comparaison traite la parole de l'autre, rapprochons ce marqueur du tour négatif [...non x mais y]. Réécrivons l'énoncé comparatif (13) en énoncé négatif (13a):

(13) La spécificité de l'époque actuelle réside moins dans la critique de la police que dans la façon dont celle-ci est formulée.

(13a) La spécificité de l'époque actuelle réside non dans la critique de la police mais dans la façon dont celle-ci est formulée.

Les deux tours mettent en relation $\mathrm{x}$ attribué à e1 et $\mathrm{y}$ attribué à $\mathrm{E} 1$; mais alors que la négation, selon la logique binaire du vrai ou faux ( $\mathrm{x}$ et $\mathrm{y}$ sont présentés comme exclusifs), rejette sans appel la parole de l'autre, la comparaison, selon la logique scalaire du plus et du moins ( $\mathrm{x}$ et y sont présentés comme non-exclusifs / compatibles), lui aménage une - petite - place. Figures différentes de l'autre dans la parole du même : tu / il a(s) tort et j'ai raison (négation) vs nous avons tous raison mais toi / lui moins que moi (comparaison d'infériorité); moi plus que toi / lui (comparaison de supériorité)...

\section{Renchérissement}

À rapprocher des deux tours précédents pour leur façon de marquer l'altérité énonciative, les renchérissements de type : [...non/pas seulement $\mathrm{x}$, (mais aussi) y].

(16) Non, l'Europe n'est pas seulement le bloc économique puissant qui pourrait aspirer à la primauté dans le monde, elle est aussi une pauvre chère vieille toute petite chose qui doit désormais protéger et revivifier ses diversités.

Sur le thème de [l'Europe est], E1 met en relation deux rhèmes $\mathrm{x}$ et $\mathrm{y}$ pour déclarer que la pertinence du premier [seulement le bloc économique puissant qui pourrait aspirer à la primauté dans le monde] qu'il attribue à e1 doit se compléter de la prise en compte $\mathrm{du}$ second [aussi une pauvre chère vieille toute petite chose...] qu'il s'attribue. Soyons plus précis encore et comparons ce tour avec la négation [... non x mais y]. Soit (16a) :

(16a) l'Europe est non le bloc économique puissant qui pourrait aspirer à la primauté dans le monde, mais une pauvre chère vieille toute petite chose.

41 La négation en non (ou ne... pas) réfute l'argument x [(l'Europe est) le bloc économique puissant qui pourrait aspirer à la primauté dans le monde.]; la négation en non seulement nie non pas $\mathrm{x}$ mais l'assertion exceptive de $\mathrm{x}$; la réfutation porte uniquement sur seulement. De sorte que E1 s'accorde avec e1 sur $\mathrm{x}$, le désaccord portant sur le fait que le thème (L'Europe) puisse avoir pour rhème le seul $\mathrm{x}$, E1 affirmant que $\mathrm{x}$ doit être complété par $\mathrm{y}^{10}$.

L'attribution de x à $\mathrm{e} 1$ se vérifie dans les tours où l'autre est explicité :

(17) Les migrants ne sont pas seulement différents, comme on voudrait le revendiquer pour eux, ils sont également différents, universellement égaux aux autres..., aux hôtes.

Comme la comparaison et à la différence de la négation, le renchérissement prend en considération la parole de l'autre pour lui faire une place; mais alors que la comparaison évalue la pertinence de deux arguments sans les relier l'un à l'autre (l'argument de e1 apparaît simplement dans sa moindre pertinence), le renchérissement pose que $\mathrm{x}$ tire toute sa pertinence d'être complété de y (l'argument de e1 apparait comme partiel). Façon d'intégrer la parole de l'autre pour mieux la dépasser, d'apparaitre comme quelqu'un qui loin de s'opposer infantilement à elle, la dote d'un 
prolongement qui lui donne toute sa valeur. E1 ne reste pas au niveau de la structure argument-contre argument : il se met en une position haute en dépassant l'apparente contrariété des arguments pour les poser en une unité dialectique supérieure ${ }^{11}$.

\section{Confirmation}

E1 peut rejeter (négation), mettre en débat (interrogation), une assertion de e1 : il peut aussi la confirmer, notamment par des adverbes modaux comme bien, évidemment, décidément, effectivement, sûrement, etc.

(18) les difficultés qu'il [Juppé] a rencontrées dans la mise en œuvre de son plan [...] ont bien suscité des interrogations sur sa crédibilité.

(19) la coopération internationale est sûrement souhaitable, à condition qu'elle ne serve pas d'alibi pour excuser nos faiblesses.

Sans entrer dans le détail du fonctionnement spécifique de chaque adverbe, je dirai que, dans ces occurrences, E1 vient ratifier l'assertion correspondante de e1, par ex. pour (18) : [les difficultés qu'il a rencontrées dans la mise en œuvre de son plan [...] ont suscité des interrogations sur sa crédibilité.]. Et ce, de différentes façons : dans (19) par ex., le mouvement de confirmation réalisé par sûrement se prolonge d'une réserve sous forme d'une condition posée [à condition qu'elle ne serve pas d'alibi pour excuser nos faiblesses]. Nous voilà tout près du marqueur dialogique à l'étude duquel nous allons maintenant procéder : la concession.

\section{La concession}

Soit la structure prototypique [w, bien sûr x mais y]. Argumentativement, E1 asserte w, s'accorde temporairement avec l'assertion de $x$ imputée à e1 qui pourrait venir en contradiction argumentative de $\mathrm{w}$, pour neutraliser par avance la conclusion qui pourrait être tirée de $\mathrm{x}$ en lui opposant $\mathrm{y}$. L'énoncé $\mathrm{x}$ est dialogique. E1 en le concédant à e1 le lui attribue :

(20) Le xxe siècle n'est décidément pas un siècle humain. Bien sûr, la démocratie a tendance à se généraliser [...]. Bien sûr, il y a eu des progrès. Mais voilà que [...] les pays développés s'acheminent vers une guérilla contre ceux qu'ils appellent des « envahisseurs ».

La concession se réalise de multiples façons (notamment par la subordination de l'élément énonciativement hétérogène : [bien que $\mathrm{x}]^{12}$ ) - pas toujours aussi clairement dialogiques que celle proposée - dans le détail de l'analyse desquelles je n'entre pas. Je ferai seulement deux remarques complémentaires :

- Le marqueur dialogique peut prendre la forme de l'adverbe oui, introduisant par là une trace de dialogalité dans un texte monologal :

(21) La réforme économique a bien évidemment échoué. Oui, le pays évolue vers le marché, mais les conséquences en sont terribles.

E1 se montre dialoguant avec un interlocuteur fictif (ici l'énonciataire E2) dont il reprend l'énoncé.

- Le marqueur dialogique, au lieu de confirmer temporairement l'assertion de e1 (mouvement de thèse), peut la poser comme seulement possible, en un mouvement d'hypothèse porté par peut-être: 
(22) Les manifestants posent peut-être des questions sans apporter de réponses, au moins eux n'attendent pas les « bonnes questions » du Front national.

\section{L'opposition}

51 Nous avons rencontré la conjonction mais - dans le tour négatif [non x mais y], dans le renchérissement [non seulement $\mathrm{x}$ mais $\mathrm{y}$ ] ainsi que dans la concession [bien sûr $\mathrm{x}$ mais y] - accompagnant un marqueur dialogique. Dans quelle mesure mais pose-t-il luimême l'altérité énonciative? Rappelons la distinction traditionnelle entre mais d'opposition rectificative (correspondant à sino en espagnol, sondern en allemand) et le mais d'opposition concessive (correspondant à pero en espagnol, aber en allemand). Seul le second est ici concerné. On connaît la description que Ducrot 1984 en donne :

[dans] les énoncés du type «p mais $q$ » [...] le premier segment (p) est présenté comme un argument pour une certaine conclusion ( $r$ ), et le second pour la conclusion inverse (1984, p. 229).

Ducrot pose que lorsque (p) est précédé de certes [certes p mais q] (tour que j'ai analysé comme concession), (p) est attribué à un autre "énonciateur", correspondant à l'«allocutaire ", mais laisse entendre (j'interprète peut-être ici un texte que je trouve flou : p. 230) que le dialogisme - Ducrot dirait la polyphonie - disparait en l'absence de certes, soit dans [p mais q]. Il illustre son analyse de l'exemple fabriqué suivant: «Je vous demande de me décrire vos skis, que je ne connais pas. Vous me répondrez peutêtre "ils sont longs mais légers " alors qu'il serait bizarre, dans la même situation, de m'annoncer « ils sont certes longs, mais ils sont légers » » (ibid.). Je propose une analyse différente sur les deux points suivants (je remplace [p mais $q]$ par [x mais y] pour harmoniser avec les analyses précédentes) :

- Si dans certaines occurrences de [x mais y], $\mathrm{x}$ n'est pas nécessairement dialogique, dans d'autres il l'est parfaitement :

(23) La morale sans le socialisme, c'est de peu d'efficacité, mais le socialisme sans la morale, ce n'est rien.

54 L'assertion de l'élément x [La morale sans le socialisme, c'est de peu d'efficacité.] est, comme lorsque la concession est formellement exprimée par certes (que l'on peut d'ailleurs ajouter ici), attribuée à e1. Altérité énonciative explicitable : [La morale sans le socialisme, c'est de peu d'efficacité, comme tout un chacun s'accorde à le penser.]. La question reste entière de savoir pourquoi x pose une altérité énonciative dans certaines occurrences de [x mais y] et non dans toutes.

55 - Ce tour est dialogique à un autre titre. Reprenons l'exemple de Ducrot [(mes skis) sont longs mais légers] : mais oppose non pas y (légers) à x (longs), mais y à (r) (que je verbalise par [donc ils sont lourds]). Or ( $r$ ) est la conclusion implicite qu'un énonciateur différent de $\mathrm{E} 1$ pourrait tirer de $\mathrm{x}^{13}$. Cette analyse vaut pour la concession [w bien sûr $\mathrm{x}$ mais y]. Prenons une autre occurrence :

(24) les grandes réformes ne peuvent réussir qu'en devenant contagieuses. Ce qui, je

le reconnais, n'est pas facile. Mais il n'existe pas d'autre voie [...]

Est attribué à e1 non seulement l'élément $\mathrm{x}$ [ce qui n'est pas facile], concédé ici explicitement par je le reconnais, mais également la conclusion implicite qu'il est supposé en tirer : [il faut donc abandonner les grandes réformes], conclusion à laquelle s'oppose [mais y]. 
57 De sorte que, au terme de cette rapide réflexion, je ne maintiens la distinction entre concession [certes $\mathrm{x}$ mais $\mathrm{y}$ ] et opposition [ $\mathrm{x}$ mais $\mathrm{y}$ ] que de ce que, alors que la concession attribue à e1 deux énoncés ( $\mathrm{x}$ et sa conclusion $\mathrm{r}$ ), certains énoncés d'opposition, tels l'exemple forgé par Ducrot [(mes skis) sont longs mais légers], ne lui attribuent que la conclusion implicite $r$.

Concession comme opposition posent que E1 prend en compte les arguments de l'autre, sait les évaluer positivement... même si c'est temporairement, pour mieux s'orienter vers une conclusion contraire.

\section{Subordination (et thématisation)}

La subordination - en tant qu'elle consiste à enchâsser une proposition dans une autre - s'avère particulièrement apte à porter le dialogisme - en tant qu'enchâssement de l'énoncé de e1 dans celui de E1 -: le discours indirect, forme prototypique de dialogisme, est introduit par que, forme prototypique de la subordination. Que, subordonnant syntaxique, offre ses services à la subordination énonciative. Cette aptitude se trouve réalisée dans la causale introduite par puisque, et par certaines subordonnées en position initiale thématisante.

\subsection{Puisque}

Ducrot (1980, p. 47-49) a montré comment puisque, à la différence de car ou parce que, introduit un énoncé « polyphonique ». Ce que j'illustre par l'occurrence suivante :

(25) (L'Occident) veut une Russie stable. Ce qui est compréhensible puisque l'instabilité de la Russie serait source de malheur pour tout le monde.

61 Par puisque, E1 pose que l'assertion de l'énoncé [l'instabilité de la Russie serait source de malheur pour tout le monde.] est le fait de e1 (ici l'instance du "ON-vérité", Berrendonner 1976/1981) et qu'il en partage le bien-fondé. Puisque sert notamment à E1 à s'appuyer sur du (prétendument) déjà asserté, par un tiers, par l'énonciataire (ou par lui-même antérieurement) à des fins de consolidation discursive. L'hétérogénéité qui fissure la parole est ici convoquée pour bétonner l'argumentation.

\subsection{Concession}

J'ai déjà mentionné le cas de [bien que $\mathrm{x}, \mathrm{y}$ ] lorsque j'ai traité de la concession. Si je le reprends, c'est qu'il est un excellent moyen de poser clairement le lien entrevu précédemment entre thématisation et dialogisme. Danon-Boileau, Meunier, Morel et Tournadre (1991), dans leur réflexion sur deux types d'intégration (syntaxique, discursive), montrent que le dialogisme de la subordonnée concessive, fort lorsque celle-ci est en position initiale : [bien que $\mathrm{x}, \mathrm{y}$ ], tend à disparaître lorsqu'elle est en position finale: $[y$, bien que $x]$, ce qu'ils mettent au compte du phénomène de la thématisation.

Dès lors qu'un segment devient thématique et cesse d'être strictement intégré au niveau de l'énoncé en cours, les opérations énonciatives qui l'affectent doivent porter trace de leur hétérogénéité ou plutôt de leur dialogisme (ibid., p. 112). 

complétive sujet en que.

\subsection{Hypothèse}

\subsection{Complétive} SV. à elle.

Les auteurs ne s'attardent pas sur les causes qui font de la thématisation un outil du dialogisme. Essayons de préciser : placé en position thématique initiale, un segment, et très précisément dans les cas de subordination, la subordonnée :

- fait le lien avec le co-texte (ce qui est dit dans le texte antérieurement, que ce soit par E1 ou par des e1) et avec le contexte (les autres discours des tiers, de l'énonciataire sous la forme du discours tenu comme du discours que E1 prête à E2 en réponse à son propre discours). En tant que tel, le segment thématique reprend du déjà asserté : résonnent en lui les voix de e1 et de E1. Il est de l'ordre du commun.

- est un tremplin à partir duquel peut s'énoncer le rhème du seul E1.

La subordination, lorsqu'elle se réalise par thématisation de l'élément subordonné, peut donc s'avérer un excellent outil de dialogisation. Outre la subordonnée de concession, je citerai, entre autres cas, celui de la subordonnée hypothétique et de la

Certains tours hypothétiques actualisent la potentialité dialogique de la subordination. L'élément subordonné s'y présente en position initiale thématique : [si x, y]. Par ex. :

(26) Si, d'ailleurs, la valeur actuelle du couple franc-mark était si avantageuse pour l'Allemagne et si nuisible pour la France, nous devrions être très déficitaires dans nos échanges avec notre puissant voisin. Or nous sommes largement excédentaires.

E1, en disant [si x], suspend l'assertion de la proposition x imputée à e1 (recul de la thèse à l'hypothèse) à la modalisation de $y$, le plus souvent pour en rejeter le bienfondé. Remarquons, qui confirme notre analyse, que e1 est parfois explicité :

(27) $\mathrm{Si}$, comme certains nous le disent, le bon taux c'est le taux zéro, il faudra expliquer à l'épargne française modeste qu'elle ne doit plus être rémunérée.

Une phrase peut être nominalisée par que, infinitisation ou nominalisation au sens restreint. Je n'aborderai ici que le premier cas, et seulement lorsque la phrase nominalisée a la fonction de sujet ${ }^{14}$. Soit [que $\left.\mathrm{x}+\mathrm{SV}\right]$ :

(28) «Les banlieues» sont présentées comme un poids mort pour la collectivité nationale. [...] Que $25 \%$ de la population active vaudaise soit au chômage est un pur scandale contre lequel nous développons des efforts acharnés.

E1, en disant [que x], « désasserte » (Danon-Boileau et al., ibid., p. 112), par le subjonctif, l'assertion de la proposition x [25 \% de la population active vaudaise est au chômage.], imputée à e1 (implicite ; contextuellement : l'agent, lui-même implicite, du verbe passif de la phrase précédente sont présentées) : en fait un dictum qui trouve son modus dans le

qui permettent à E1 de prendre en compte la parole de e1, même si c'est pour s'opposer 


\section{Conclusion} importance au concept de dialogisme et s'attacher à la description de ses marques. Quels autres tel discours convoque-t-i ${ }^{15}$ ? quelle place (en termes quantitatifs comme en termes qualitatifs) leur accorde-t-il ? Quel type de marqueur tend-il à privilégier? Dis-moi quels sont tes autres et comment tu les traites et je te dirai qui tu es ${ }^{16} \ldots$ Audelà, le dialogisme ne permet-il pas de revisiter la linguistique textuelle ? On remarque que, aux trois niveaux emboîtés de l'énoncé, du paragraphe et du texte lui-même, le dialogisme tend à marquer les éléments initiaux. Par exemple, au niveau textuel : les titres s'avèrent fréquemment frappés de dédoublement énonciatif, rarement les conclusions. $\mathrm{Au}$ niveau phrastique: le dialogisme affecte particulièrement les subordonnées à l'initiale. Thématisation de la parole de l'autre : ne peut-on concevoir que la production discursive, aux trois niveaux du texte, du paragraphe et de l'énoncé, va du dialogique (le thème) au monologique (le rhème) : de [E1(e1)] à [E1], du commun au propre (illusoire bien sûr) ? Cet ordre ne nous montre-t-il pas la façon dont le même tente de se dégager de l'autre, ce en quoi consisterait la production discursive ellemême?

74 - L'importance quantitative du dialogisme n'atteste-t-elle pas que le sujet parlant est, plus encore qu'un "homme de paroles", un homme de dialogues? Même si c'est réducteur, on peut dire avec Bakhtine que le dialogisme est la façon pour le texte monologal de dire l'interaction verbale dans un genre du discours qui ne peut l'accueillir sous sa forme dialogale :

Dans les limites d'un énoncé, le locuteur (ou le scripteur) pose des questions, y répond lui-même, oppose des objections que lui-même réfute, etc. Ces phénomènes ne sont que la simulation conventionnelle de l'échange verbal (Bakhtine 1979/1984, p. 278).

On n'échappe pas au dialogue : humain, trop humain. Voilà qui a des conséquences d'importance au niveau discursif: le dialogue pose fondamentalement - à travers la réversibilité de la personne - l'égalité des interlocuteurs. Le dialogisme se présente certes comme une hiérarchisation ([E1(e1)]). Mais citer la parole de l'autre, fût-ce pour la rejeter sans appel dans le tour le plus agonal, c'est se placer avec l'autre sur un pied d'égalité interlocutive. Le dialogue que permet le dialogisme place le sujet parlant dans la mêlée des discours à égalité de droits et de devoirs. Vouloir se placer au-dessus de cette mêlée c'est péché d'orgueil et, nous l'avons vu, peine perdue. Ce que ne dit pas le texte biblique, c'est que Dieu châtia les hommes pour l'édification de la Tour de Babel non seulement par la multiplication des langues mais aussi par l'imposition du dialogisme...

76 - Bienheureux châtiment en tout cas : le principe dialogique, l'impossible clôture du discours sur lui-même, c'est dans la perspective bakhtinienne le signe du joyeux inachèvement de l'humain, de la possibilité de l'histoire qui libère, à l'opposé de l'achevé de toute éternité du divin, de l'ordre monologique qui asservit. On ne peut qu'être frappé à la lecture des textes de Bakhtine d'une part, de ceux des membres de l'équipe Pêcheux d'autre part, de la différence de ton, plus précisément encore de climat, dans la prise en compte de l'altérité constitutive du discours à lui-même: enthousiasme et allégresse ici, dramatisation voire désespoir tragique là (l'humour de 
Michel Pêcheux...). On peut certes en rendre compte par la différence de niveau de l'analyse : Bakhtine n'avait pas rencontré le grand Autre de l'inconscient qui construit le sujet en totale extériorité à lui-même. Il me semble cependant y avoir plus : et si les analyses de l'école Pêcheux s'inscrivaient historiquement dans le courant du « sérieux unilatéral et à signification unique » (Bakhtine 1963/1970, p. 195) qui n'en finit pas de faire son deuil du cogito cartésien, alors que celles de Bakhtine s'inscrivent dans l'autre courant: celui du "comique-sérieux ", " pénétré de sensibilité carnavalesque » (ibid., 125) qui fait du relatif et du rire la mesure de toute chose?

\section{BIBLIOGRAPHIE}

Adamson, S. (1994), « Subjectivity in narration: empathy and echo », in Subjecthood and subjectivity, Paris: Ophrys, p. 193-208.

Authier-Revuz, J. (1982), « Hétérogénéité montrée et hétérogénéité constitutive ; éléments pour une approche de l'autre en discours », DRLAV 26, p. 91-151.

Authier-Revuz, J. (1984), « Hétérogénéités énonciatives », Langages 73, p. 98-111.

Authier-Revuz J., 1995, Ces mots qui ne vont pas de soi, Paris : Larousse.

Bakhtine, M. (1929/1977), Le marxisme et la philosophie du langage, Paris : Minuit.

Bakhtine, M. (1934/1978), « Du discours romanesque », in Esthétique et théorie du roman, Paris : Gallimard, Tel, p. 83-233.

Bakhtine, M. (1963/1970), Problèmes de la poétique de Dostoïevski, Lausanne : L'âge d'homme.

Bakhtine, M. (1979/1984), « Les genres du discours », in Esthétique de la création verbale, Paris : Gallimard, p. 265-308.

Bally, C. (1934/1965), Linguistique générale et linguistique française, Berne : Francke.

Berrendonner, A. (1976/1981), « Le fantôme de la vérité », in Eléments de pragmatique linguistique, Paris : Minuit, p. 35-73.

Bouguerra, T. \& J. Bres (1995), « Le texte journalistique et son écriture », in C. Détrie, M. Masson \& B. Verine (eds) Pratiques textuelles, Langue et praxis : Praxiling, Montpellier III, p. 251-284.

Bres, J. (1993), Récit oral et production d'identité sociale, Montpellier III : Praxiling.

Bres, J. (1998), « Entendre des voix : de quelques marqueurs dialogiques en français », in Bres J., Legrand R., Madray F. et P. Siblot (éds), L'autre en discours, (à paraître).

Danon-Boileau, L. (1987), Le sujet de l'énonciation, Paris : Ophrys.

Danon-Boileau, L., Meunier, A., Morel, M.-A. et N. Tournadre (1991), « Intégration discursive et intégration syntaxique », Langages 104, 111-128.

Ducrot, O. (1984), « Esquisse d'une théorie polyphonique de l'énonciation », in Le dire et le dit, Paris : Minuit, p. 171-233.

Ducrot, O. et al. (1980), Les mots du discours, Paris : Minuit. 
Guentchéva, Z. (1995), « L'énonciation médiatisée et les mécanismes perceptifs », in J. Bouscaren, J.-J. Franckel \& S. Robert (éds) Langues et langage, Paris : PUF, p. 301-315.

Haillet, P. (1995), Le conditionnel dans le discours journalistique : essai de linguistique descriptive, Québec : Bref.

Haillet, P. (1998), « Le conditionnel d'altérité énonciative et les formes du discours rapporté dans la presse écrite ", Pratiques (à paraître).

Pêcheux, M. (1975), Les vérités de la Palice, Paris : Maspéro.

Pêcheux, M. (1990), L'inquiétude du discours, Paris : Editions des cendres.

Todorov, T. (1981), Mikhail Bakhtine. Le principe dialogique, suivi d'Ecrits du cercle de Bakhtine, Paris : Minuit.

Vion, R. (1994), « De l'hétérogénéité des instances énonciatives », Cahiers du français contemporain 1, 227-246.

\section{NOTES}

1. Je ne prends pas partie dans la querelle de paternité qui entoure certains textes. J'emploie le patronyme Bakhtine comme métonymie de Cercle de Bakhtine.

2. Bakhtine pose un 3e type de dialogisme : « les rapports de dialogue entre le sujet parlant et sa propre parole » (Bakhtine 1963/1970 : 212). Je ne traiterai pas de cette dimension autodialogique.

3. Certaines d'entre elles ont fait l'objet de description (notamment Ducrot 1984, Authier-Revuz 1992), dans des perspectives et des cades théoriques différents des miens.

4. Cet article fait partie d'un travail sur le thème de L'autre en discours, qui a donné matière à une première publication complémentaire de celle-ci (Bres 1998), dans laquelle se trouvent développés les cadres théoriques ainsi que l'analyse de certains marqueurs dialogiques (interrogation, extraction, détachement).

5. Le conditionnel est en français le signifiant par lequel se réalise la catégorie du médiatif (cf. Guentcheva 1995).

6. Autre fonctionnement dialogique de la négation restrictive : E1 sélectionne un seul rhème dans un ensemble d'au moins deux rhèmes prêté à e1.

7. Remarquons que l'inverse - l'inscription de E1 sous l'argument déclaré de moindre pertinence et l'inscription de e1 sous l'argument déclaré de plus grande pertinence - n'est possible qu'avec une forme verbale au passé qui introduit une distance énonciative (fragmentation de E1 en deux instances : autodialogisme), et réclame l'explicitation des sources énonciatives. Soit en modifiant (13) en (13a) :

(13a) La spécificité de l'époque actuelle réside moins dans la critique de la police comme je l'ai affirmé/ *l'affirme/ que dans la façon dont celle-ci est formulée, comme le dit le ministre de l'intérieur.

Cette inversion du principe discursif de valorisation du même/dévalorisation de l'autre, théoriquement possible donc, n'est pas actualisée dans le corpus des 29 occurrences de comparaison d'inégalité recueilli, ce qui me semble significatif.

8. Ce qui engage peut-être à reconsidérer la structure de ce type d'énoncé, qui serait non pas : Th $+\mathrm{Rh}(\mathrm{x})+\mathrm{Rh}(\mathrm{y})$ mais $\mathrm{Th}+\mathrm{Rh}(\mathrm{x})+\mathrm{Th}(\mathrm{y})$.

9. C'est également la prédication qui me semble expliquer la transformation de [... plus / davantage $x$ que $y$ ] en [plus/davantage que $y, x$ ] :

(15) [...] jusqu'à devenir (le rap) mieux qu'un reflet, le véritable moyen d'expression d'une jeunesse multiraciale et multiculturelle. 
L'élément rhématique $\mathrm{x}$ est placé en position finale, place la plus rhématisante en français.

10. Il serait également intéressant de comparer, notamment du point de vue des effets de sens, le renchérissement et la comparaison d'égalité.

11. N'est-il pas significatif que ce tour soit l'outil de prédilection du "penseur de la complexité » E. Morin, dans l'article sélectionné pour le corpus?

12. Je renvoie à l'article de Danon-Boileau, Meunier, Morel et Tournadre (1991, p. 113) pour l'analyse de la différence des fonctionnements dialogiques dans la coordination et la subordination.

13. En contexte narratif, mais convoque la voix du narrataire. Soit la séquence suivante, extraite de Le Mas Théotime de H. Bosco :

«Je l'ai prise par le bras et je l'ai emmenée [...]. Je lui ai lâché le bras mais elle m'a suivi docilement."

A la différence de et [et elle m'a suivi en silence], mais présuppose un énoncé-réponse du narrataire qui est présenté comme tirant de l'élément $x$ la conclusion (r) : [elle s'est enfuie]. Et c'est à cette conclusion que le narrateur s'oppose par [mais elle m'a suivi].

14. En position d'objet, la nominalisation phrastique rejoint la question plus générale du discours rapporté indirect (Pierre prétend que Sophie le trompe), qui pose des problèmes spécifiques de dialogisme liés au sémantisme du verbe introducteur, que je choisis de ne pas aborder ici.

15. Ou essaie-t-il d'effacer. Un seul exemple : on sait que Benveniste a pris grand soin à ne pas se référer à la théorisation de Guillaume - par ex. à la question de Ricœur, lors d'un débat, lui demandant de se situer par rapport à celle-ci, il ne sera pas fait réponse (Benveniste 1967/1974, p. 236, in Problèmes de linguistique générale II, Paris : Gallimard) -, à (essayer de) gommer de ses textes toute trace d'intertextualité avec la psychomécanique. Peine perdue : chassé par la porte du discours rapporté, le discours de l'autre revient, par ex. par la fenêtre de la négation : «Du signe à la phrase il n'y a pas transition [...]» (1969/1974, p.65, ibid.). Une oreille avertie en psychomécanique ne peut manquer d'entendre dans transition un mot de Guillaume dans son questionnement de la transition de la langue au discours.

16. «La manière individuelle dont l'homme construit son discours est, pour une part considérable, déterminée par la sensation personnelle qu'il a du mot de l'autre et par les moyens qu'il a d'y réagir » (Bakhtine 1963/1970, p. 229).

\section{AUTEUR}

\section{JACQUES BRES}

Praxiling

UPRESA 5475, Discours, textualité et production de sens

Université Montpellier III 